\title{
Impacts of ivermectin mass drug administration for onchocerciasis on mosquito populations of Ogun state, Nigeria
}

\author{
Olaitan Olamide Omitola ${ }^{1 *}$ (D), Cynthia Uchechukwu Umunnakwe' , Adedotun Ayodeji Bayegun', \\ Samuel Akinjide Anifowose ${ }^{1}$, Hammed Oladeji Mogaji ${ }^{2} \mathbb{D}$, Akinola Stephen Oluwole ${ }^{3}$, \\ Simon Nnayere Odoemene ${ }^{4}$, Taiwo Sam Awolola ${ }^{5}$, Adebola Adedoyin Osipitan ${ }^{6}$, \\ Sammy Olufemi Sam-Wobo ${ }^{1}$ and Uwem Friday Ekpo ${ }^{1 *}$ (D)
}

\begin{abstract}
Background: The impact of single-dose mass drug administration (MDA) of ivermectin for onchocerciasis on mosquito populations was investigated in Ogun State, Nigeria.

Methods: Indoor and outdoor collection of mosquitoes was carried out in two intervention (IC) and two control communities (CC) at three different periods: pre-MDA (baseline), 2-3 days after MDA and 13-14 days after MDA. The density and parity rate of female Anopheles and Culex mosquitoes were determined and compared. Environmental and climatic data of study locations were obtained to perform generalized linear model analysis.
\end{abstract}

Results: A total of 1399 female mosquitoes were collected, including 1227 Anopheles and 172 Culex mosquitoes. There was a similar magnitude of reduction in the indoor density of Anopheles by $29 \%$ in the IC and CC $2-3$ days post-MDA but the reduction in indoor parity rate was significantly higher $(p=0.021)$ in the $I C$, reducing by more than $50 \%$. In the IC, observation of a significant reduction at 2-3 days post-MDA was consistent for both the indoor density (1.43 to 1.02 ) and indoor parity rate $(95.35 \%$ to $44.26 \%$ ) of Anopheles mosquitoes. The indoor parity rate of Anopheles remained significantly reduced (75.86\%) 13-14 post-MDA. On the other hand, the indoor density of Culex increased from 0.07 to 0.10 at 2-3 days post-MDA while the indoor parity rate of Culex did not change. The outdoor density of Anopheles in the IC increased ( $p=0.394$ ) from 0.58 to 0.90 at 2-3 days post-MDA; a similar observation was consistent for the outdoor density (2.83 to 3.90) and outdoor parity rate (70.59\% to $97.44 \%$ ) of Culex, while the outdoor parity rate of Anopheles reduced from 85.71 to $66.67 \%$ at $2-3$ days post-MDA. A generalized linear model showed that ivermectin MDA significantly caused a reduction in both the indoor density $(p<0.001)$ and indoor parity rate $(p=0.003)$ of Anopheles in the IC.

Conclusion: Ivermectin MDA resulted in the reduction of both the survival and density of Anopheles mosquitoes. This has strong implications for malaria transmission, which depends strongly on vector survival.

Keywords: Ivermectin, Endectocide, Mosquito, Vector control, Nigeria

*Correspondence: olaitanomitola@gmail.com; ufekpo@hotmail.com

${ }^{1}$ Department of Pure and Applied Zoology, Federal University of Agriculture, Abeokuta, Ogun, Nigeria

Full list of author information is available at the end of the article

\section{Introduction}

Mosquitoes alone account for $17 \%$ of the estimated global burden of infectious diseases, and every year, malaria which is transmitted by the Anopheles mosquito, causes the deaths of more than 400,000 people and incapacitates

c) The Author(s) 2021. This article is licensed under a Creative Commons Attribution 4.0 International License, which permits use, sharing, adaptation, distribution and reproduction in any medium or format, as long as you give appropriate credit to the original author(s) and the source, provide a link to the Creative Commons licence, and indicate if changes were made. The images or other third party material in this article are included in the article's Creative Commons licence, unless indicated otherwise in a credit line to the material. If material is not included in the article's Creative Commons licence and your intended use is not permitted by statutory regulation or exceeds the permitted use, you will need to obtain permission directly from the copyright holder. To view a copy of this licence, visit http://creativeco mmons.org/licenses/by/4.0/. The Creative Commons Public Domain Dedication waiver (http://creativecommons.org/publicdomain/ zero/1.0/) applies to the data made available in this article, unless otherwise stated in a credit line to the data. 
another 200 million for days [1]. In Nigeria, malaria accounts for about $11 \%$ maternal mortality, $25 \%$ infant mortality, 30\% under-5 mortality, $60 \%$ outpatient visits and $30 \%$ hospital admissions [2]. To avert the deaths and morbidity caused by malaria and other mosquito-borne diseases, vector control has been adopted as the mainstay of control strategies. Current methods involve the deployment of insecticides to eliminate biting or resting mosquitoes by mounting long-lasting insecticidal nets (LLINs) where the mosquito may land when trying to reach the person sleeping under the net and spraying walls inside the house where blood-fed mosquitoes will often rest [3]. However, mosquitoes are still able to escape these strategies because they bite when people are not under LLINs or because they rest or feed outside the sprayed house.

Interest in the development of interventions that are capable of targeting mosquitoes that can escape the current strategies for vector control is emerging. The adoption of ivermectin, used in the treatment of filarial worm infections, has been proposed to address limitations of the current malaria control tools such as residual transmission [4]. Residual transmission occurs when malaria continues to be transmitted, despite the effective implementation of available vector control tools, as a result of mosquitoes adapted to biting outside the time of the day when LLINs and indoor residual spraying can protect people. Ivermectin is a systemic endectocide, which, when administered to humans or animals, is also toxic to mosquitoes that feed on the treated vertebrate host. This provides an opportunity to eliminate mosquitoes that may escape or survive existing vector control interventions since all female mosquitoes must obtain blood meals for the development of their eggs [3].

Studies have reported the ability of ivermectin to reduce the life span and vectorial capacity of Anopheles sp. that feed on treated humans $[4,5]$. Although it is shown that Anopheles mosquitoes captured from villages after ivermectin MDA have reduced lifespans when monitored in the laboratory, this does not adequately represent the mass effect on mosquito populations under real-life settings. There is a need to evaluate how the effect of ivermectin on mosquito lifespan impacts the density or abundance of the mosquito population in a locality.

Besides, generating local evidence of the mosquitocidal potentials of ivermectin mass drug administration will assist in effective targeting and optimized delivery of the drug in the context of vector control in endemic areas. Findings suggest the extent of the mosquitocidal effect of ivermectin may vary within the anopheline group [4]. Hence, it is important to understand how the local vector population of a geographical area, which comprises a unique diversity of primary and secondary vector species, is affected during ivermectin mass drug administration. In this study, we investigated the impacts of the annual round of ivermectin MDA, used for the control of onchocerciasis and lymphatic filariasis (LF), on local mosquito vector populations in the communities of Odeda local government area (LGA) in Ogun State, Nigeria.

\section{Methods}

\section{Study area}

This study was carried out in Odeda Local Government Area (LGA) of Ogun State, Nigeria, between August and September 2018. Odeda LGA is endemic for onchocerciasis and LF, where community-directed treatment with ivermectin (CDTI) is being implemented annually through mass drug administration (MDA) with single-dose administration of $150-200 \mu \mathrm{g} / \mathrm{kg}$ ivermectin (Mectizan $^{\circledR}$, Merck \& Co Inc.). Treatment coverage was between 66 and 80\% in the LGA between 2013 and 2017 according to the ivermectin therapeutic coverage records of the Ogun State Ministry of Health (SMoH). Four communities-Kugba-Ajagbe $\left(7.36102^{\circ} \mathrm{N}, 3.59830^{\circ} \mathrm{E}\right)$ and Amini $\left(7.23509^{\circ} \mathrm{N}, 3.74935^{\circ} \mathrm{E}\right)$ as intervention communities and Olofin $\left(7.41974^{\circ} \mathrm{N}, 3.64086^{\circ} \mathrm{E}\right)$ and Gbagba $\left(7.44530^{\circ} \mathrm{N}, 3.63307^{\circ} \mathrm{E}\right)$ as control communities-were selected for the study. The intervention communities have been receiving ivermectin MDA rounds consistently from 2013 to 2017, whereas the control communities have no records of ivermectin treatment (Fig. 1). The four communities are a part of the sparsely dispersed settlements in the border region between Ogun and Oyo States of Nigeria. Each community is located about $3 \mathrm{~km}$ from the nearest villages in the surrounding area.

\section{Study design}

The cross-sectional study involved the collection of mosquito samples from each community at three different sampling intervals in Fig. 2.

In each community, 15 houses were enumerated for the collection of indoor-resting mosquitoes using systematic selection, beginning with the village head's resident as the reference house. Where consent could not be obtained, the next consenting household was selected. Three outdoor locations, where inhabitants commonly gather in the evenings, were also selected in each community for outdoor mosquito collection [6]. Sampling intervals were spaced to allow sufficient time for recoil in the mosquito populations and minimize the effect of the mosquito collection on the population density. MDA with ivermectin was carried out by the Ogun State neglected tropical diseases (NTD) control programme on 3 and 10 September 2018 in Kugba-Ajagbe and Amini, respectively. The 


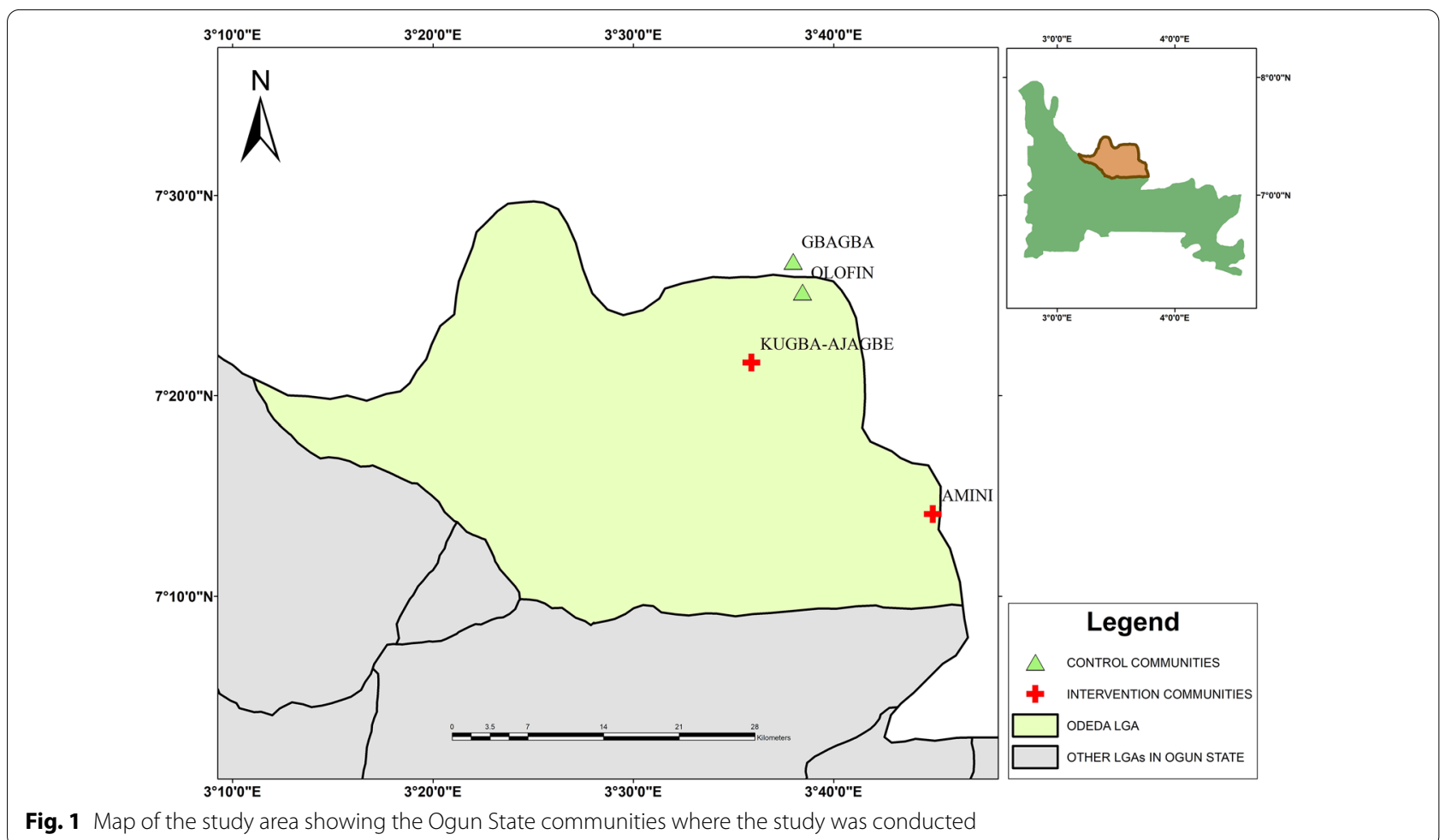

Fig. 1 Map of the study area showing the Ogun State communities where the study was conducted

Matched pair of

intervention and control

communities

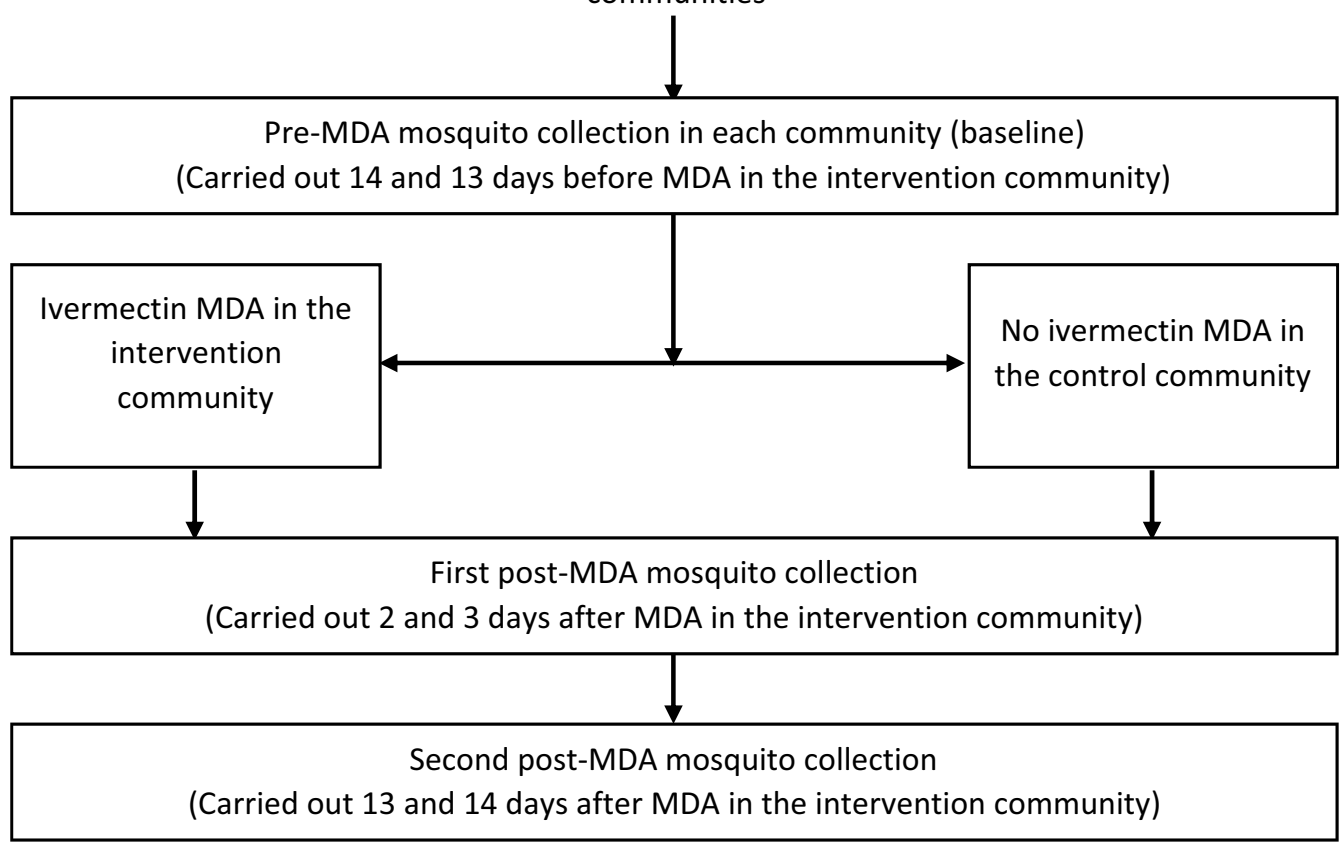

Fig. 2 The study design for mosquito collection 
surrounding villages of both the intervention and control communities received ivermectin MDA during the study.

\section{Collection, identification and dissection of mosquitoes}

Mosquitoes were collected indoors between 05.00 and $09.00 \mathrm{~h}$ in the morning by adapting the pyrethrum spray catch (PSC) method previously described [7], with modifications including the use of the commercial aerosol Raid $^{\circledR}$ (containing $0.250 \%$ allethrin, $0.150 \%$ tetramethrin, 0.015\% deltamethrin and $99.585 \%$ inert ingredients). This was sprayed in each room by two collectors, who returned after $10 \mathrm{~min}$ to retrieve the knocked-down mosquitoes. CDC Miniature Light Traps (Model 512; John W. Hock Co., Gainesville, FL, USA) were used as a convenient and cheap alternative to human-landing catch (HLC) for outdoor collection of mosquitoes because of the ethical concerns and labour involved in HLC $[8,9]$. During each mosquito collection visit to a community, three light traps were set up in three outdoor locations at about $18.00 \mathrm{~h}$ at dusk and retrieved at about $06.00 \mathrm{~h}$ at dawn. A bottle, containing a mixture of yeast and sugar solution, was hung near each trap as $\mathrm{CO}_{2}$ bait. Mosquitoes were collected from the rooms and traps into labelled Petri dishes and the mosquito samples were transported to the laboratory at the Department of Pure and Applied Zoology. The female mosquitoes were sorted and identified to genus level using morphological keys [10]. All the female Anopheles and Culex mosquitoes collected were dissected for age grouping (parity status) based on the ovarian tracheoles $[6,11]$.

\section{Entomological parameters}

Mosquito population characteristics were measured in terms of density (abundance) and parity rate (age structure). The indoor density of mosquitoes was calculated using the formula below:

$$
\text { Density }=\frac{\text { Number of female mosquitoes collected }}{\text { Number of rooms } \times \text { Number of nights }} \text {. }
$$

The outdoor density of mosquito was calculated using the formula below:

$$
\text { Density }=\frac{\text { Number of female mosquitoes collected }}{\text { Number of traps } \times \text { Number of nights }} .
$$

Parity rate was also calculated as shown below:

$$
\begin{aligned}
& \text { Parity rate }(\%) \\
& =\frac{\text { Number of parous female mosquitoes }}{\text { Total number of female mosquitoes }} \times 100 .
\end{aligned}
$$

\section{Determination of ivermectin MDA coverage}

MDA coverage was determined 3-4 weeks after MDA in the intervention communities using the coverage survey builder (CSB) protocol and a questionnaire developed by the WHO [12]. The number of households surveyed was calculated with the CSB, estimated at 15 households per community. The questionnaire was administered, and respondents were shown a sample of the drug package to facilitate recall during the interviews. Interviews were conducted by translating the questionnaire into the local language. Every member of the selected household was interviewed, regardless of their age or eligibility for MDA. Where a household member was absent or too young to respond personally, the household head or an available adult member of the household responded on their behalf.

\section{Remote-sensing climatic and environmental data}

Geographic coordinates of the communities were obtained with a portable GPS receiver $\left(\mathrm{eTrex}^{\circledR} 10\right.$, Garmin $^{\mathrm{TM}}$ International, Olathe, KS, USA). Rainfall and vegetation index data were collected as possible confounders on mosquito populations during the study. Data for precipitation and the normalized difference vegetation index (NDVI) were sourced from two open-access satellite imagery databases: EarthExplorer [13] and Precipitation Estimation from Remotely Sensed Information using Artificial Neural Networks (PERSIANN), respectively [14]. For each mosquito collection visit, available data for 14 days up to the collection date were retrieved since mosquito development lasts for about 5-14 days.

\section{Data analyses}

Secondary data from remote sensing resources were processed using the HDF-EOS to GeoTIFF (HEG) Conversion Tool (Raytheon Co., Riverdale, MD, USA) and ArcGIS 10.3 (ESRI Incorp., Redlands, CA, USA) was used to extract raster values for the relevant climatic and environmental variables. These data, together with primary data from mosquito collection and coverage survey, were inputted into a Microsoft Office Excel spreadsheet (Microsoft Corp., Redmond, WA, USA) and imported into IBM SPSS Statistics (IBM Corp., Somers, NY) for statistical analyses. Data were subjected to descriptive statistics to present frequency, total, mean and percentage tables. Mann-Whitney $U$ test and chi-square goodness-of-fit test were used to compare mosquito abundance between sampling intervals within a community and between study areas. Pearson chi-square test was used to compare mosquito parity rates between the sampling intervals and to compare MDA coverage between communities. Ivermectin exposure was graded into (1) early post-MDA, (2) late Post-MDA and (3) pre-MDA 
zero exposure, based on the relative degree to which MDA was expected to impact the mosquito populations at the different sampling intervals. A generalized linear model was applied to determine the effects of MDA alone as well as the effects of MDA alongside other factors on the density and parity rate of the mosquitoes in the intervention communities. In all the instances of statistical analyses, a $p$-value $<0.05$ was used to determine statistical significance.

\section{Results}

\section{Composition and distribution of the mosquito samples collected}

A total of 1399 mosquitoes were collected across the study communities: 1227 (87.7\%) were Anopheles sp. and $172(12.3 \%)$ were Culex sp. Of the 1227 Anopheles sp. collected, 975 (79.4\%) were from the control communities while 109 (63.4\%) of the 172 Culex sp. were collected from the intervention communities. Among the Anopheles sp. collected, a total of 1109 (90.4\%) and $118(9.6 \%)$ were indoor and outdoor resting mosquitoes, respectively. Similarly, among the Culex sp. a total of 135 (78.5\%) and 37 (21.55) were outdoor and indoor resting mosquitoes, respectively (Table 1 ).

\section{Comparison of mosquito density between sampling intervals}

The density of mosquitoes collected indoors at the sampling intervals in the intervention and control communities is shown in Fig. 3. Before MDA, the indoor density of Anopheles sp. was significantly higher $(p=0.043)$ in the control communities (7.20) than in the intervention communities (1.43). At 2-3 days post-MDA, the density reduced in both the intervention and control communities by a factor of $29.07 \%$ and $28.61 \%$, without a significant difference between both reductions $(p=0.561)$.

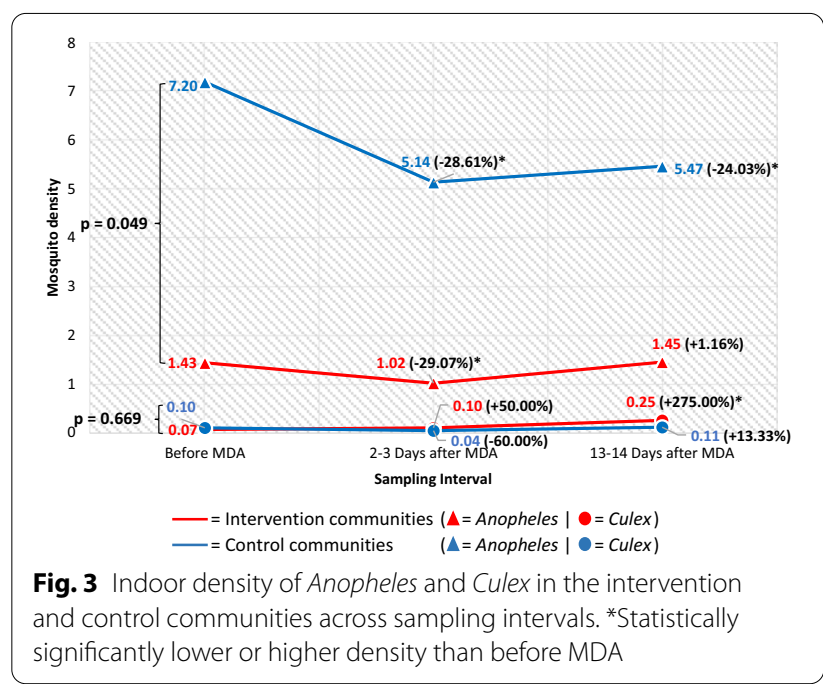

In the intervention communities, the indoor density of Anopheles sp. reduced significantly $(1.02, p=0.039)$ 2-3 days post-MDA but increased 13-14 days postMDA $(1.45, p=0.939)$ compared to pre-MDA. In the control communities, this was 5.14 and 5.47 at 2-3 days and 13-14 days post-MDA, both significantly $(p<0.001)$ lower than before MDA. The difference between the indoor density of Culex sp. in the control and intervention communities before MDA was not statistically significant $(0.10$ vs $0.07, p=0.752)$. In the intervention communities, this increased $2-3$ days $(0.10, p=0.527)$ and $13-14$ days post-MDA $(0.25, p=0.012)$ compared to pre-MDA. In the control communities, this reduced $2-3$ days post-MDA $(0.04, p=0.257)$ and increased $13-14$ days post-MDA $(0.11, p=0.849)$ compared to pre-MDA.

In Fig. 4, the densities of mosquitoes collected outdoors at the sampling intervals are shown. Before the

Table 1 Descriptive statistics of the mosquito samples collected

\begin{tabular}{|c|c|c|c|c|c|c|}
\hline & \multicolumn{2}{|l|}{ Intervention } & \multicolumn{2}{|l|}{ Control } & \multicolumn{2}{|l|}{ Total } \\
\hline & No. of mosquitoes & Parous mosquitoes & No. of mosquitoes & Parous mosquitoes & No. of mosquitoes & Parous mosquitoes \\
\hline & $N(\%)$ & $N$ (parity) & $N(\%)$ & $N$ (parity) & $N(\%)$ & $N$ (parity) \\
\hline \multicolumn{7}{|l|}{ Anopheles sp. } \\
\hline Indoor & $234(92.9)$ & $175(74.79)$ & $875(89.7)$ & $792(90.51)$ & 1109 (90.4) & $967(87.20)$ \\
\hline Outdoor & $18(7.1)$ & $12(66.67)$ & $100(10.3)$ & $68(68.00)$ & $118(9.6)$ & $80(67.80)$ \\
\hline Total & 252 & 187 & 975 & 860 & 1227 & 1047 \\
\hline \multicolumn{7}{|l|}{ Culex sp. } \\
\hline Indoor & $25(22.9)$ & $24(96.00)$ & $12(19.0)$ & $11(91.67)$ & $37(21.5)$ & $35(94.60)$ \\
\hline Outdoor & $84(77.1)$ & $72(85.71)$ & $51(81.0)$ & $41(80.39)$ & $135(78.5)$ & $113(83.70)$ \\
\hline Total & 109 & 96 & 63 & 52 & 172 & 148 \\
\hline Overall total & 361 & 283 & 1038 & 912 & 1399 & $1195(85.42)$ \\
\hline
\end{tabular}




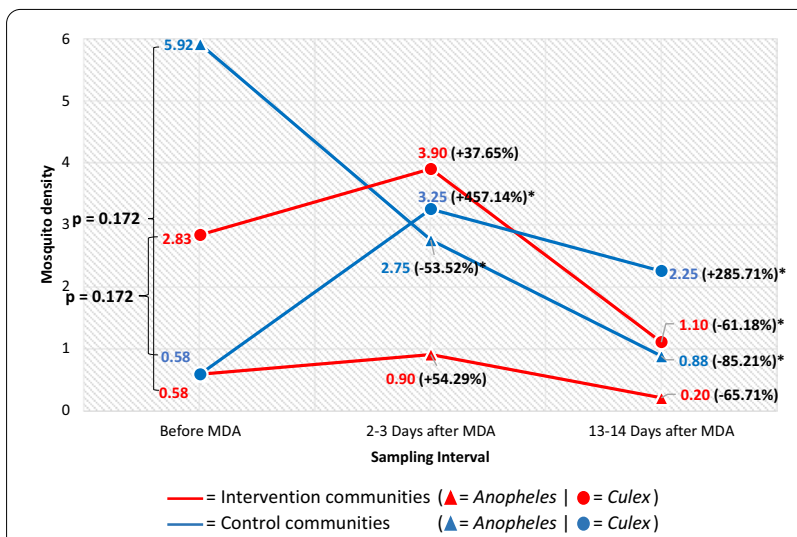

Fig. 4 Outdoor density of Anopheles and Culex in the intervention and control communities across sampling intervals. *Statistically significantly lower or higher density than before MDA
MDA, the difference in the outdoor density of Anopheles sp. between the control and intervention communities was not statistically significant (5.92 vs $0.58, p=0.465)$. In the intervention communities, this increased 2-3 days post-MDA $(0.90, p=0.394)$ and reduced $13-14$ days post-MDA $(0.20, p=0.160)$ compared to before MDA. In the control communities, this reduced significantly $2-3$ days $(2.75, p=0.001)$ and $13-14$ days post-MDA $(0.88, p<0.001)$ compared to pre-MDA. The difference between the outdoor density of Culex sp. in the control and intervention communities before MDA was also not statistically significant (0.58 vs 2.83, $p=0.053)$. In the intervention communities, this increased 2-3 days post-MDA (3.90, $p=0.173)$ and reduced significantly 13-14 days post-MDA $(1.10, p=0.005)$ compared to pre-MDA. In the control communities, the density at $2-3$ days (3.25) and 13-14 days post-MDA (2.25), was significantly higher than pre-MDA $(p<0.001$ and $p=0.001$ respectively).

\section{Comparison of mosquito parity rate between sampling intervals}

Figure 5 illustrates the parity rate of the mosquitoes collected indoors. The percentage reduction in the indoor parity rate of Anopheles sp. 2-3 days post-MDA was significantly higher $(p=0.021)$ in the intervention $(53.58 \%)$ than in the control communities (0.67\%). In the intervention communities, the indoor parity rate of Anopheles sp. reduced significantly $2-3$ days $(44.26 \%, p<0.001)$ and 13-14 days post-MDA (75.86\%, $p=0.001)$ compared to $95.35 \%$ before MDA. In the control communities, this reduced 2-3 days post-MDA $(89.31 \%, p=0.910)$ and increased 13-14 days post-MDA $(92.68 \%, p=0.303)$ compared to $89.92 \%$ before MDA. Similarly, the indoor parity rate of Culex sp. in the intervention communities

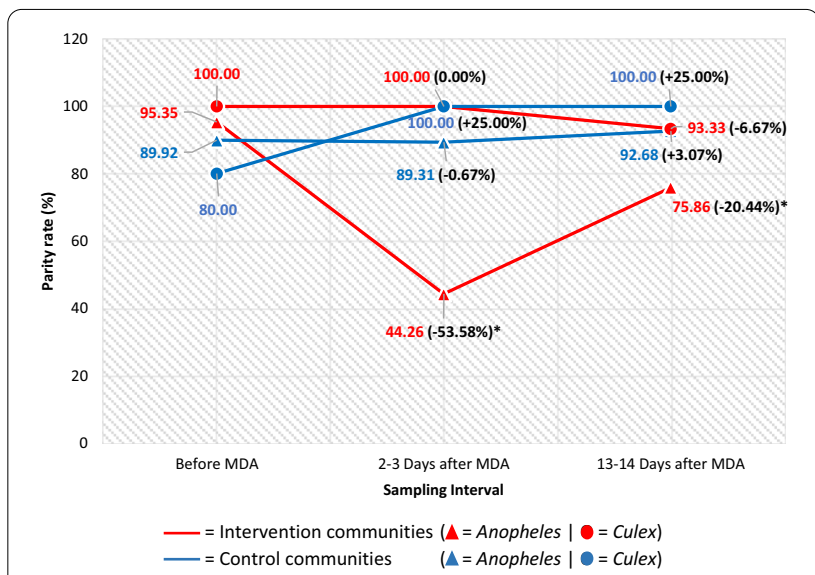

Fig. 5 Parity rate of Anopheles and Culex collected indoors in the intervention and control communities. *Statistically significantly lower or higher parity rate than before MDA

was $100.00 \%$ pre-MDA and did not change significantly 2-3 days (100.00\%) and 13-14 days post-MDA (93.33\%, $p=0.789)$. In the control communities, this increased $2-3$ days $(100.00 \%, p=0.714)$ and $13-14$ days post-MDA $(100.00 \%, p=0.500)$ compared to $80.00 \%$ before MDA.

In Fig. 6, the parity rate of Anopheles sp. collected outdoors in the intervention communities reduced 2-3 days (66.67\%, $p=0.392)$ and $13-14$ days post-MDA $(0.00 \%$, $p=0.083$ ) from $85.71 \%$ before MDA. In the control communities, this increased $2-3$ days $(77.27 \%, p=0.288)$ and $13-14$ days post-MDA $(100.00 \%, p=0.044)$ from $61.97 \%$ before MDA. The outdoor parity rate of Culex sp. in the intervention communities was higher at 2-3 days $(97.44 \%, p=0.004)$ and $13-14$ days post-MDA $(90.91 \%$, $p=0.170)$ compared to $70.59 \%$ before MDA. In the control communities, this increased $2-3$ days (76.92\%,

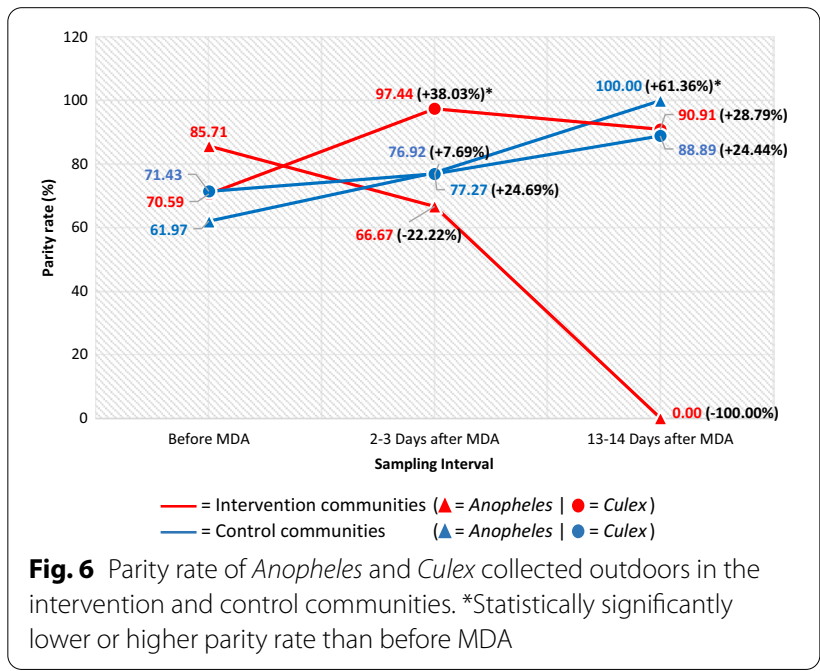


$p=0.556)$ and $13-14$ days post-MDA $(88.89 \%, p=0.307)$ compared to $71.43 \%$ pre-MDA.

\section{MDA coverage and changes in mosquito populations} in the intervention communities

The coverage evaluation survey showed that MDA coverage was significantly $(p=0.024)$ higher in Amini than Kugba-Ajagbe (Table 2). MDA coverage in the two intervention communities, as well as changes in the density and parity rate of Anopheles and Culex populations, are also shown in Table 2.

\section{Data for rainfall and vegetation index}

In all the communities, average rainfall progressively increased from the period before MDA to the period after MDA (Table 3). The normalized difference vegetation index (NDVI) also increased progressively in KugbaAjagbe and Gbagba. However, changes in the NDVI in Amini and Olofin were contrasting and not similar.

Table 2 Ivermectin MDA coverage and mosquito population changes in the intervention communities

\begin{tabular}{|c|c|c|c|c|}
\hline & & Kugba-Ajagbe & Amini & $p$-value \\
\hline \multicolumn{5}{|c|}{ № of eligible persons surveyed } \\
\hline \multicolumn{2}{|c|}{ Female } & 32 & 23 & \\
\hline \multicolumn{2}{|l|}{ Male } & 29 & 18 & \\
\hline \multicolumn{2}{|c|}{ Total } & 61 & 41 & \\
\hline \multicolumn{5}{|c|}{ №. of persons treated (\%) } \\
\hline \multicolumn{2}{|c|}{ Female } & $17(68.0)$ & $14(51.9)$ & \\
\hline \multicolumn{2}{|l|}{ Male } & $8(32.0)$ & $13(48.1)$ & \\
\hline \multicolumn{2}{|l|}{ Total coverage } & $25(41.0)$ & $27(65.9)$ & $0.024^{*}$ \\
\hline \multicolumn{5}{|l|}{ Anopheles sp. } \\
\hline \multirow[t]{3}{*}{ Indoor density } & 14-13 days before MDA & 1.97 & 0.90 & \\
\hline & 2-3 days post-MDA & $1.70(p=0.446)$ & $0.33(p=0.005)^{\dagger}$ & \\
\hline & 13-14 days post-MDA & $1.93(p=0.926)$ & $0.97(p=0.789)$ & \\
\hline \multirow[t]{3}{*}{ Outdoor density } & 14-13 days before MDA & 1.00 & 0.17 & \\
\hline & 2-3 days post-MDA & $0.83(p=0.763)$ & $1.00(0.068)$ & \\
\hline & 13-14 days post-MDA & $0.33(p=0.157)$ & 0.00 & \\
\hline \multirow[t]{3}{*}{ Indoor parity rate } & 14-13 days before MDA & 96.61 & 92.59 & \\
\hline & 2-3 days post-MDA & $45.10(p<0.001)^{\dagger}$ & $40.00(p=0.002)^{\dagger}$ & \\
\hline & 13-14 days post-MDA & $67.24(p<0.001)^{\dagger}$ & $93.10(p=0.667)$ & \\
\hline \multirow[t]{3}{*}{ Outdoor parity rate } & 14-13 days before MDA & 83.33 & 100.00 & \\
\hline & 2-3 days post-MDA & $80.00(p=0.727)$ & $50.00(p=0.600)$ & \\
\hline & 13-14 days post-MDA & $0.00(p=0.107)$ & - & \\
\hline \multicolumn{5}{|l|}{ Culex sp. } \\
\hline \multirow[t]{3}{*}{ Indoor density } & 14-13 days before MDA & 0.13 & 0.00 & \\
\hline & 2-3 days post-MDA & $0.07(p=0.414)$ & 0.13 & \\
\hline & 13-14 days post-MDA & $0.23(p=0.366)$ & 0.27 & \\
\hline \multirow[t]{3}{*}{ Outdoor density } & 14-13 days before MDA & 1.50 & 4.17 & \\
\hline & 2-3 days post-MDA & $5.33(p<0.001)^{\dagger}$ & $1.75(p=0.036)^{\dagger}$ & \\
\hline & 13-14 days post-MDA & $1.50(p=1.000)$ & $0.50(p=0.001)^{\dagger}$ & \\
\hline \multirow[t]{3}{*}{ Indoor parity rate } & 14-13 days before MDA & 100.00 & - & \\
\hline & 2-3 days post-MDA & 100.00 & 100.00 & \\
\hline & 13-14 days post-MDA & 100.00 & 87.50 & \\
\hline \multirow[t]{3}{*}{ Outdoor parity rate } & 14-13 days before MDA & 88.89 & 64.00 & \\
\hline & 2-3 days post-MDA & $96.88(p=0.395)$ & $100.00(p=0.073)$ & \\
\hline & 13-14 days post-MDA & $88.89(p=0.765)$ & $100.00(p=0.436)$ & \\
\hline
\end{tabular}

*Difference between total MDA coverage is statistically significant

${ }^{\dagger}$ Density or parity rate is statistically significantly higher or lower than before MDA 
Table 3 Data for rainfall and vegetation index across the sampling intervals

\begin{tabular}{llll}
\hline & $\begin{array}{c}14-13 \text { days } \\
\text { before MDA }\end{array}$ & 2-3 days post-MDA & $\begin{array}{l}13-14 \text { days post- } \\
\text { MDA }\end{array}$ \\
\hline $\begin{array}{llll}\text { Rainfall }(\mathrm{mm}) \\
\text { Kugba-Ajagbe }\end{array}$ & 0.2760 & $2.2273\left(0.028^{*}\right)$ & $4.5480\left(0.018^{*}\right)$ \\
Amini & 0.8827 & $3.9467\left(0.040^{*}\right)$ & $4.7820(0.071)$ \\
Olofin & 0.2539 & $1.4413(0.093)$ & $5.3407\left(0.012^{*}\right)$ \\
Gbagba & 0.7887 & $4.7653\left(0.033^{*}\right)$ & $5.2660\left(0.018^{*}\right)$ \\
Vegetation index & & & \\
Kugba-Ajagbe & 0.2535 & $0.5298\left(<0.001^{*}\right)$ & $0.5862\left(<0.001^{*}\right)$ \\
Amini & 0.1872 & $0.4779\left(<0.001^{*}\right)$ & $0.4156\left(<0.001^{*}\right)$ \\
Olofin & 0.2269 & $0.1118\left(<0.001^{*}\right)$ & $0.5318\left(<0.001^{*}\right)$ \\
Gbagba & 0.2535 & $0.5756\left(<0.001^{*}\right)$ & $0.6360\left(<0.001^{*}\right)$ \\
\hline
\end{tabular}

*Rainfall or vegetation index is statistically significantly lower or higher than before MDA

Table 4 Effect of ivermectin MDA alone on mosquito density and parity rate in the intervention communities

\begin{tabular}{llclc}
\hline & Anopheles sp. & $p$-value & Culex sp. & $p$-value \\
\hline $\begin{array}{llcll}\text { Density } \\
\text { Indoor }\end{array}$ & Intercept & 0.170 & Intercept & $<0.001$ \\
& Exposure to MDA & 0.663 & Exposure to MDA & $0.005^{*}$ \\
Outdoor & Intercept & 0.249 & Intercept & $<0.001$ \\
& Exposure to MDA & 0.826 & Exposure to MDA & $0.041^{*}$ \\
Parity rate & & & & \\
Indoor & Intercept & $<0.001$ & Intercept & $<0.001$ \\
& Exposure to MDA & $<0.001^{*}$ & Exposure to MDA & 0.517 \\
Outdoor & Intercept & $<0.001$ & Intercept & $<0.001$ \\
& Exposure to MDA & $<0.001^{*}$ & Exposure to MDA & 0.104 \\
\hline
\end{tabular}

*Statistically significant effect on mosquito density or parity rate

Effects of ivermectin MDA, rainfall and vegetation index on the mosquito density and parity rate in the intervention communities

In Table 4, the generalized linear model showed that ivermectin MDA alone had a significant effect on the parity rate of indoor $(p<0.001)$ and outdoor $(p<0.001)$ Anopheles populations in the intervention communities but no significant effects on density. Ivermectin MDA alone also showed a significant effect on the density of indoor $(p=0.005)$ and outdoor $(p=0.041)$ Culex populations in the intervention communities but no significant effects on parity rate.

In Table 5, the generalized linear model showed that ivermectin MDA had a significant effect on the density $(p<0.001)$ and parity rate $(p=0.003)$ of indoor
Anopheles populations in the intervention communities. Rainfall also showed a significant effect $(p<0.001)$ on the density of indoor Anopheles populations in the intervention communities whereas the vegetation index showed a significant effect $(p=0.031)$ on the density of the outdoor Anopheles population in the intervention communities.

\section{Discussion}

Before ivermectin MDA, the densities of the indoor and outdoor populations of Anopheles mosquitoes were more than five times lower in the intervention communities than in the control communities. This difference between the indoor populations was statistically significant. In contrast, there was no statistically significant difference in the densities of Culex between the two study areas. A similar observation was reported in a longitudinal study carried out in northeastern Tanzania, where a decline in the density of Anopheles was observed while the abundance of Culex mosquitoes remained unaffected $[15,16]$. Derua et al. [17] attributed this observation in Tanzania to the use of ivermectin for the control of onchocerciasis and LF in the area for $>10$ years. In our study, the intervention communities had received ivermectin MDA for at least 5 years while there was no MDA in the control communities within the same period. Although we did not conduct a longitudinal investigation, the baseline Anopheles density differences observed between the intervention and control communities could be hypothetically attributed to long-term ivermectin mass drug administration for onchocerciasis and LF control.

A reduction in the daily survival rate is recognised as a primary effect of ivermectin MDA on malaria vectors in insectary-based studies [18]. In our field-based evaluation, this translated to a reduction in the abundance of malaria vectors after MDA. In the intervention communities, the indoor density of Anopheles mosquitoes reduced 2-3 days after ivermectin MDA, and a rebound became noticeable after 2 weeks. These changes in the malaria vector abundance followed a consistent pattern in the two intervention communities. In contrast, the abundance of Culex sp. increased significantly in the intervention communities 2-3 days after ivermectin MDA. Studies have shown that unlike Anopheles sp., the other mosquito vectors are not readily susceptible to the concentrations of ivermectin found in human blood after MDA with the currently recommended safe dosage of the drug $[17,19]$. This indicates that factors other than the MDA likely account for the observed reduction in the indoor density of Culex sp. in one of the intervention communities.

Interestingly, we observed a reduction in the density of Anopheles mosquitoes in the control communities 
Table 5 Generalized linear model showing effects of MDA, rainfall and vegetation index in the intervention communities

\begin{tabular}{|c|c|c|c|c|c|c|c|c|}
\hline & \multicolumn{4}{|l|}{ Indoor } & \multicolumn{4}{|l|}{ Outdoor } \\
\hline & $B$ & SE & $\mathrm{Cl}$ & $p$-value & B & SE & $\mathrm{Cl}$ & $p$-value \\
\hline \multicolumn{9}{|l|}{ Anopheles sp. } \\
\hline \multicolumn{9}{|l|}{ Density } \\
\hline Intercept & 0.134 & 0.5810 & -1.004 to 1.273 & 0.319 & -6.375 & 2.8019 & $\begin{array}{r}-11.86 \text { to } \\
(-0.883)\end{array}$ & 0.029 \\
\hline Exposure to $\mathrm{MDA}^{\dagger}$ & & & & $<0.001^{*}$ & & & & 0.094 \\
\hline Rainfall & -0.868 & 0.2240 & -1.307 to $(-0.429)$ & $<0.001^{*}$ & 0.838 & 0.4699 & -0.084 to 1.759 & 0.075 \\
\hline Vegetation index & 2.971 & 2.1644 & -1.271 to 7.213 & 0.170 & 24.235 & 11.213 & 2.258 to 46.211 & $0.031^{*}$ \\
\hline \multicolumn{9}{|l|}{ Parity rate } \\
\hline Intercept & 5.011 & 0.3920 & 4.242 to 5.779 & $<0.001$ & 5.985 & 2.0793 & 1.910 to 10.060 & 0.048 \\
\hline Exposure to $\mathrm{MDA}^{\dagger}$ & & & & $0.003^{*}$ & & & & 0.457 \\
\hline Rainfall & -0.127 & 0.1510 & -0.423 to 0.169 & 0.399 & -0.333 & 0.3487 & -1.017 to 0.350 & 0.339 \\
\hline Vegetation index & -1.732 & 1.4594 & -4.593 to 1.128 & 0.235 & -5.799 & 8.3210 & -22.108 to 10.510 & 0.486 \\
\hline \multicolumn{9}{|l|}{ Culex sp. } \\
\hline \multicolumn{9}{|l|}{ Density } \\
\hline Intercept & -1.801 & 0.5176 & -2.816 to $(-0.787)$ & 0.216 & 0.487 & 1.1259 & -1.720 to 2.694 & 0.996 \\
\hline Exposure to $\mathrm{MDA}^{\dagger}$ & & & & 0.099 & & & & 0.650 \\
\hline Rainfall & -0.025 & 0.2189 & -0.454 to 0.404 & 0.910 & 0.302 & 0.4486 & -1.181 to 0.577 & 0.501 \\
\hline Vegetation index & -0.817 & 1.7724 & -4.290 to 2.657 & 0.645 & 3.857 & 4.3361 & -4.641 to 12.356 & 0.374 \\
\hline \multicolumn{9}{|l|}{ Parity rate } \\
\hline Intercept & 4.313 & 0.1723 & 3.976 to 4.651 & $<0.001$ & 4.406 & 0.5203 & 3.387 to 5.426 & $<0.001$ \\
\hline Exposure to $\mathrm{MDA}^{\dagger}$ & & & & 0.434 & & & & 0.442 \\
\hline Rainfall & 0.034 & 0.0729 & -0.109 to 0.176 & 0.644 & -0.093 & 0.1990 & -0.483 to 0.297 & 0.639 \\
\hline Vegetation index & 1.115 & 0.5900 & -0.042 to 2.271 & 0.059 & -1.045 & 1.9234 & -4.814 to 2.725 & 0.587 \\
\hline
\end{tabular}

SE standard error, Cl 95\% confidence interval

*Statistically significant effect on mosquito density or parity rate

+ Categorical variable

at a magnitude similar to the reduction observed in the intervention communities after MDA. This investigation was carried out in a study area where the spatial scale may make it possible for both humans and mosquitoes to move between, at least, one of the intervention communities and its pair-matched control community [20]. In addition, the proximity of the control communities to the surrounding villages, where the annual ivermectin MDA programme proceeded normally during the study may contribute to the observed reduction in the density of Anopheles in the control communities. However, it is also notable that the study area is located within the forest ecozone of Nigeria, where the contamination of vector populations between the control communities and the other surrounding villages may be limited by natural environmental barriers. In addition, the absence of a significant change in the parity rate of Anopheles mosquitoes in the control communities 2-3 days after ivermectin MDA contradicts the possible effect of contamination from the surrounding villages where MDA was conducted. The factors responsible for this observation in the control communities are not clear at the moment, which calls for further investigation.

The short-lived reduction in the indoor density of Anopheles sp. in the two intervention communities is similar to observations from previous studies on the life span or survival rate of Anopheles mosquitoes captured from villages treated with ivermectin. In a study carried out in Senegal, Liberia and Burkina Faso, reduction in the daily survival rate of An. gambiae was only observable within the first week after MDA [18]. Also, a clinical trial in Burkina Faso showed that mortality increased for up to 7 days in An. gambiae, which were fed with blood from individuals treated with a single dose of ivermectin [5]. It is generally believed that the recommended dose of ivermectin for onchocerciasis and LF control programmes will not have long-lasting lethal effects on malaria vectors $[19,21]$.

On the other hand, disruption of the age structure of Anopheles mosquitoes can last up to 3 weeks after ivermectin MDA [18, 20]. In our study, the proportion of parous (older) female Anopheles of the indoor population 
also remained significantly reduced by $>20 \%$ in the intervention communities 2 weeks after MDA. This significant shift to a younger population of female Anopheles sp. has important implications for malaria transmission because the older or parous female mosquitoes are commonly the infectious vectors [19]. It has been indicated that the impact on the mosquito population age structure may be the main mechanism by which ivermectin MDA affects malaria transmission [4]. An ivermectin-treated blood meal kills most of the infectious mosquitoes leaving behind a population predominated by young nulliparous mosquitoes, which require some time to become infectious [19].

Importantly, the reduction in the indoor density of Anopheles mosquitoes by $29.07 \%$ in the intervention communities 2-3 days after MDA was statistically significant. However, our models suggest that ivermectin MDA did not show a clear effect on the indoor density of the Anopheles mosquitoes. Using the relative exposure to ivermectin during the three sampling intervals, the model indicated that ivermectin exposure alone showed no significant effect on the indoor density of Anopheles sp. but demonstrates a significant effect when other factors are considered. On the other hand, ivermectin exposure showed a clear effect on the parity rate of the indoor Anopheles mosquitoes in the intervention communities in our generalized linear models. Hence, although ivermectin MDA may not have a long-lasting impact on the density of Anopheles sp., it reduced the proportion of parous older mosquitoes, which are critical for the transmission of malaria in the localities.

Our findings suggest that ivermectin MDA will have more profound effects on the density and parity rate of the Anopheles populations at higher MDA coverage. In Amini, where the coverage of ivermectin MDA was significantly higher, the density and parity rate of the indoor Anopheles population reduced by $63 \%$ and $57 \%$ respectively 2-3 days after MDA. These reductions in the density and parity rate were higher and statistically significant compared to Kugba-Ajagbe where the MDA coverage was lower. Therefore, higher MDA coverage in the intervention communities has the potential to enhance the mosquitocidal effects of ivermectin compared to the current observations. Interestingly, there was a prolonged reduction in the indoor density and parity rate of Anopheles mosquitoes in Kugba-Ajagbe, where a higher proportion of female individuals was treated with ivermectin. This finding is corroborated by the trial conducted in Burkina Faso, where the lethal effects of ivermectin were stronger and more prolonged in the Anopheles mosquitoes that fed on female individuals compared to the males because the female individuals have higher adipose tissue mass, which aids the accumulation of ivermectin and also acts as a slow-release mechanism for the drug [5].

Ivermectin did not show an effect on the outdoor populations of malaria vectors in our study. Unlike the indoor Anopheles populations, the outdoor density of Anopheles mosquitoes in the intervention communities increased 2-3 days after ivermectin MDA. Although the parity rate of the outdoor Anopheles population decreased progressively after MDA, our models showed no significant effects of ivermectin MDA on either the density or parity rate of outdoor Anopheles populations. In addition, variations in the density and parity rate of the outdoor Anopheles populations in the two intervention communities did not show corresponding patterns as observed for the indoor populations. In this study, samples of the outdoor mosquito populations were collected using the CDC light trap method. Several limitations of this method for outdoor sampling of malaria vectors have been highlighted in different settings, including the possibility of underestimating the density of host-seeking mosquitoes, which might have affected the number of outdoor mosquito samples collected during this study [22]. Although our $\mathrm{CDC}$ light traps were augmented with improvised $\mathrm{CO}_{2}$ baits to improve performance, the baits may have operated for a limited period during mosquito collection. Therefore, our findings may provide limited information on the impact of ivermectin MDA on the outdoor populations of malaria vectors in the communities where the study was carried out.

In Nigeria, sibling species of the An. gambiae complex such as An. gambiae s.s. and An. arabiensis are recognised as the principal vectors of malaria, and emerging shifts toward zoophily, outdoor biting and exophily have been reported, indicating an increasing challenge of residual transmission of malaria in the country [23, 24]. Although the morphological identification carried out in our study does not include information on the species of Anopheles mosquitoes collected, previous investigations carried out in locations close to our study area showed that the Anopheles samples collected comprised only the known vectors of malaria in Nigeria $[25,26]$. Our findings support the need for further investigation of ivermectin MDA as a complementary tool for malaria control. Ivermectin is distributed annually or semi-annually through the mass drug administration programmes for the control of onchocerciasis and lymphatic filariasis. This provides an opportunity to synergize the existing NTD programmes with an ivermectin MDA programme targeted at malaria elimination. The Ivermectin Roadmap outlines the processes needed for the deployment of ivermectin as a vector control tool by 2024, including the need to establish a target population and the level of community uptake required [27]. Our study has provided 
evidence highlighting the importance of ivermectin therapeutic coverage in achieving an effective reduction in Anopheles mosquitoes as well as the transmission of malaria.

\section{Conclusions}

The abundance of Anopheles mosquitoes was significantly lower in the intervention communities where the annual ivermectin MDA round has been ongoing for a long period. The density of malaria vectors was reduced in the two intervention communities after ivermectin MDA for onchocerciasis and LF. Although the density of Anopheles mosquitoes may rebound quickly after singledose ivermectin MDA, disruption of the age structure and its implication for malaria transmission will likely last for a longer time. Overall, a high MDA coverage targeting a high proportion of female inhabitants in a community will maximize the benefits of ivermectin as a control tool for malaria vectors.

\footnotetext{
Abbreviations

MDA: Mass drug administration; LF: Lymphatic filariasis; LLIN: Long-lasting insecticidal net; IRS: Indoor residual spraying; LGA: Local government area; WHO: World Health Organization; DDT: Dichlorodiphenyltrichloroethane; CDD: Community-directed distributor.
}

\section{Acknowledgements}

We thank the staff of the Ogun State Ministry of Health's NTD unit as well as Odeda LGA's NTD unit and Primary Healthcare Directorate for the support to access the communities and for providing the population data, MDA records and other relevant data for the study. We appreciate the community heads, members, CDDs and staff of the frontline primary healthcare facilities for their cooperation throughout the study.

\section{Authors' contributions}

$\mathrm{OOO}$ and UFE conceived the study and participated in fund seeking for the research. OOO, ASO, TSA, AAO, SOS and UFE designed the study. OOO, CUE, $A A B, S A A$ and SNA participated in the acquisition, curating, and analysis of data. OOO, HOM, AAO, SOS and UFE interpreted the data for the study. TSA, AAO, SOS, and UFE supervised the study. OOO, CUE, AAB, SAA, HOM, SNO, ASO, and UFE contributed to the drafting and revising of the manuscript. All authors read and approved the final manuscript.

\section{Funding}

The study was funded by the Association of African Universities (https://www. aau.org/) through the 2017 Small Grants for Graduate Theses and Dissertations (no. PC/06). The funders had no role in the study design, data collection and analysis, interpretation of data, decision to publish or writing of the manuscript.

\section{Availability of data and materials}

The datasets for climatic and environmental variables generated and analysed during the current study are publicly available in the remote sensing data repositories cited. The primary datasets generated and analysed are available from the corresponding authors on reasonable request.

\section{Declarations}

\section{Ethics approval and consent to participate}

The study was approved by the Ethics Review Committee of the Department of Planning, Research and Statistics of the Ogun State Ministry of Health, Abeokuta (registration number: HPRS/381/276). Permission was also obtained from the Department of Primary Healthcare of Odeda LGA and the primary healthcare facilities for each of the selected communities. Communities assented to the study through verbal consents from the village heads and community leaders. The research purpose and protocol were explained in the local language in a meeting with the community leaders. Houses and households were enrolled for the study after consents were obtained from the head of the household.

\section{Consent for publication}

Not applicable.

\section{Competing interests}

The authors declare that they have no competing interests.

\section{Author details}

${ }^{1}$ Department of Pure and Applied Zoology, Federal University of Agriculture, Abeokuta, Ogun, Nigeria. ${ }^{2}$ Department of Animal and Environmental Biology, Federal University Oye Ekiti, Ekiti, Nigeria. ${ }^{3}$ COUNTDOWN Consortium, Sightsavers Nigeria Country Office, Kaduna, Nigeria. ${ }^{4}$ Department of Biological Sciences, Adeleke University, Ede, Osun, Nigeria. ${ }^{5}$ Molecular Entomology and Vector Control Research Laboratory, Public Health Division, Nigeria Institute of Medical Research, Lagos, Nigeria. ${ }^{6}$ Department of Crop Protection, Federal University of Agriculture, Abeokuta, Ogun, Nigeria.

Received: 16 December 2020 Accepted: 1 April 2021

Published online: 20 April 2021

\section{References}

1. ISGlobal. Mosquitoes: world's deadliest animal. 2017. https://www.isglo bal.org/en/new/-/asset_publisher/JZ9fGljXnWpl/content/mosquito-elanimal-mas-letal-del-mundo. Accessed 10 Sept 2018.

2. Federal Ministry of Health. Nigeria malaria indicators survey 2015: final report. Abuja and Maryland: National Malaria Elimination Programme, National Population Commission, National Bureau of Statistics, and ICF International. 2016. https://dhsprogram.com/pubs/pdf/MIS20/MIS20.pdf. Accessed 16 June 2019.

3. Steketee RW, Ter Kuile FO. Editorial commentary: ivermectin as a complementary strategy to kill mosquitoes and stop malaria transmission? Clin Infect Dis. 2014;60(3):366-8.

4. MPAC. Ivermectin for malaria transmission control: technical consultation meeting report. Malaria Policy Advisory Committee, World Health Organisation. 2016. https://www.who.int/malaria/mpac/mpac-sept2016-inver mectin-session9.pdf?ua=1. Accessed 21 Dec 2017.

5. Ouédraogo AL, Bastiaens GJ, Tiono AB, Guelbéogo WM, Kobylinski KC, Ouédraogo A, et al. Efficacy and safety of the mosquitocidal drug ivermectin to prevent malaria transmission after treatment: a double-blind, randomized, clinical trial. Clin Infect Dis. 2015;60(3):357-65.

6. Abeyasinghe RR, Yapabanadara AM, Kusumawathie PHD, Perera D, Peiris BSL, Hewavitharane HMP, et al. Guidelines for entomological surveillance of malaria vectors in Sri Lanka. Anti Malaria Campaign, Sri Lanka. 2009. http://amc.health.gov.lk/images/Publication\%20Repository/SOP/Revis ed_Guidelines_for_Entomological_surveillance.pdf. Accessed 21 July 2018.

7. WHO. Manual on practical entomology in malaria/prepared by the WHO Division of Malaria and Other Parasitic Diseases. World Health Organization. 1995. https://apps.who.int/iris/handle/10665/42481. Accessed 21 July 2018.

8. Githeko AK, Service MW, Mbogo CM, Atieli FA, Juma FO. Sampling Anopheles arabiensis, A. gambiae sensu lato and A. funestus (Diptera: Culicidae) with CDC light-traps near a rice irrigation area and a sugarcane belt in western Kenya. Bull Entomol Res. 1994;84(03):319.

9. Sriwichai P, Karl S, Samung Y, Sumruayphol S, Kiattibutr K, Payakkapol A, et al. Evaluation of CDC light traps for mosquito surveillance in a malariaendemic area on the Thai-Myanmar border. Parasites Vectors. 2015;8:636.

10. Gillett JD. Common African mosquitoes and their medical importance. London: John Swain and Co.; 1972.

11. Atieli F. Examination of ovaries by tracheal distension to determine parity. In: Benedict MQ, editor. MR4 - methods in Anopheles Research. Atlanta: CDC; 2007. 
12. WHO. Coverage evaluation surveys for preventive chemotherapy: field guide for implementation. World Health Organization. 2016. https:// www.ntdsupport.org/sites/default/files/uploads/docs/resources/Cover age\%20Evaluation\%20Guidelines\%20Final\%20Draft_Nov\%202016.pdf. Accessed 22 July 2018.

13. Earth Explorer. United States Geological Survey (USGS). https://earthexplo rer.usgs.gov/. Accessed 29 Apr 2019

14. Center for Hydrometeorology and Remote Sensing (CHRS) Data Portal. University of California, Irvine. http://chrsdata.eng.uci.edu/. Accessed 29 Apr 2019.

15. Simonsen PE, Pedersen EM, Rwegoshora RT, Malecela MN, Derua YA, Magesa SM. Lymphatic filariasis control in Tanzania: effect of repeated mass drug administration with ivermectin and albendazole on infection and transmission. PLoS Negl Trop Dis. 2010;4(6):e696.

16. Meyrowitsch DW, Pedersen EM, Alifrangis M, Scheike TH, Malecela MN, Magesa SM. Is the current decline in malaria burden in sub-Saharan Africa due to a decrease in vector population? Malar J. 2011;10:188.

17. Derua YA, Kisinza WN, Simonsen PE. Differential effect of human ivermectin treatment on blood-feeding Anopheles gambiae and Culex quinquefasciatus. Parasites Vectors. 2015:8:130.

18. Alout H, Krajacich BJ, Meyers Jl, Grubaugh ND, Brackney DE, Kobylinski $\mathrm{KC}$, et al. Evaluation of ivermectin mass drug administration for malaria transmission control across different West African environments. Malar J. 2014;13:417.

19. Chaccour CJ, Kobylinski KC, Bassat Q, Bousema T, Drakeley C, Alonso P. Ivermectin to reduce malaria transmission: a research agenda for a promising new tool for elimination. Malar J. 2013;12:153.

20. Kobylinski KC, Sylla M, Chapman PL, Sarr MD, Foy BD. Ivermectin mass drug administration to humans disrupts malaria parasite transmission in Senegalese villages. Am J Trop Med Hyg. 2011;85:3-5.
21. Bockarie MJ, Hii JL, Alexander ND, Bockarie F, Dagoro H, Kazura JW, et al. Mass treatment with ivermectin for filariasis control in Papua New Guinea: impact on mosquito survival. Med Vet Entomol. 1999;13:120-3.

22. Mboera LEG, Knols BGJ, Braks MAH, Takken W. Comparison of carbon dioxide-baited trapping systems for sampling outdoor mosquito populations in Tanzania. Med Vet Entomol. 2000;14(3):257-63.

23. Okorie PN, McKenzie FE, Ademowo OG, Bockarie M, Kelly-Hope L. Nigeria Anopheles vector database: an overview of 100 years' research. PLoS ONE. 2011;6(12):e28347.

24. Durnez L, Coosemans M. Residual transmission of malaria: an old issue for new approaches. In: Manguin S, editor. Anopheles mosquitoes-new insights into malaria vectors. Online: IntechOpen. 2013. https://www. intechopen.com/books/anopheles-mosquitoes-new-insights-into-malar ia-vectors/residual-transmission-of-malaria-an-old-issue-for-new-appro aches. Accessed 13 Jan 2021.

25. Adeleke MA, Mafiana CF, Idowu AB, Adekunle MF, Sam-Wobo SO. Mosquito larval habitats and public health implications in Abeokuta, Ogun State, Nigeria. Tanzan J Health Res. 2008;10(2):103-7.

26. Adeleke MA. Population dynamics of indoor sampled mosquitoes and their implication in disease transmission in Abeokuta, south-western Nigeria. J Vector Borne Dis. 2010;2010(47):33-8.

27. The Ivermectin Roadmappers. A roadmap for the development of ivermectin as a complementary malaria vector control tool. Am J Trop Med Hyg. 2020;102(2s):3-24.

\section{Publisher's Note}

Springer Nature remains neutral with regard to jurisdictional claims in published maps and institutional affiliations.
Ready to submit your research? Choose BMC and benefit from:

- fast, convenient online submission

- thorough peer review by experienced researchers in your field

- rapid publication on acceptance

- support for research data, including large and complex data types

- gold Open Access which fosters wider collaboration and increased citations

- maximum visibility for your research: over 100M website views per year

At BMC, research is always in progress.

Learn more biomedcentral.com/submissions 